\title{
Stabilizer Formalism for Generalized Concatenated Quantum Codes
}

\author{
Yun-Jiang Wang ${ }^{* \dagger}$, Bei Zeng ${ }^{\ddagger}$, Markus Grassl ${ }^{\S}$ and Barry C. Sanders* \\ *Institute for Quantum Science and Technology, University of Calgary, \\ Calgary, Alberta T2N 1N4, Canada, Email: sandersb@ucalgary.ca \\ $\dagger$ State Key Laboratory of Integrated Services Networks, Xidian University, \\ Xi'an, Shaanxi 710071, China Email: yunjiang.w@gmail.com \\ ‡Department of Mathematics and Statistics, University of Guelph, \\ Guelph, Ontario N1G 2W1, Canada, Email: zengb@uoguelph.ca

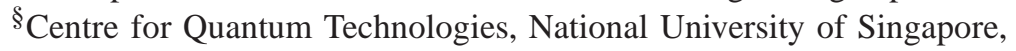 \\ 3 Science Drive 2, Singapore 117543, Singapore, Email: Markus.Grassl@nus.edu.sg
}

\begin{abstract}
The concept of generalized concatenated quantum codes (GCQC) provides a systematic way for constructing good quantum codes from short component codes. We introduce a stabilizer formalism for GCQCs, which is achieved by defining quantum coset codes. This formalism offers a new perspective for GCQCs and enables us to derive a lower bound on the code distance of stabilizer GCQCs from component codes parameters, for both non-degenerate and degenerate component codes. Our formalism also shows how to exploit the error-correcting capacity of component codes to design good GCQCs efficiently.
\end{abstract}

\section{INTRODUCTION}

Error-correcting codes are necessary to overcome restrictions in computation and communication due to noise, but developing algorithms for finding 'good' codes is generically an intractable problem and evidently the central question of coding theory. 'Good codes' are special in that they have good trade-off among rate, distance, encoding and decoding costs, thereby reducing requisite space and time resources.

In classical settings, constructing generalized concatenated codes, which incorporate multiple outer codes concatenated with multiple inner codes, is a promising approach for realizing good trade-off among those parameters [1], [2]. Recently, generalized concatenation has been introduced into the quantum scenario, providing a systematic way to construct good quantum codes with short component codes [3], [4].

The stabilizer formalism plays a central role in almost all branches of quantum information science, especially in quantum coding theory. Stabilizer codes, which are quantum analogues of classical linear codes, form the most important class of quantum error-correcting codes (QECCs) [5], [6]. The stabilizer formalism serves not only a role analogous to the classical parity-check matrix, but also takes a role analogous to the classical generator matrix during the decoding and encoding procedures [5], [7]. However, the stabilizer formalism for generalized concatenated quantum codes (GCQCs) has not been investigated in much detail previously, and the understanding of GCQCs is still far from satisfactory compared to their classical counterparts.

In this work we introduce the stabilizer formalism for GCQCs, thereby providing a new perspective for the GCQC framework as well as a powerful and systematic technique for constructing good stabilizer codes. By using our stabilizer formalism, we derive a lower bound on the achievable distance for GCQCs. Moreover, our stabilizer formalism for GCQCs clarifies how to exploit the error-correcting capacity of component codes to improve the performance of the resultant codes efficiently.

\section{Generalized concatenAted Stabilizer codes}

A qudit is a quantum system modeled by a $q$-dimensional Hilbert space $\mathbb{C}^{q}$, where $q$ is a prime power. A stabilizer (or additive) quantum code encoding $k$ qudits into an $n$-qudit system, with minimum distance $d$, is denoted by $\llbracket n, k, d \rrbracket_{q}$.

\section{A. Idea of generalized concatenated quantum codes}

A concatenated stabilizer code is constructed from two component quantum codes: an outer code $A$ with parameters $\llbracket N, K, D \rrbracket_{Q}$ and an inner code $B$ with parameters $\llbracket n, k, d \rrbracket_{q} 1$ such that $Q=q^{k}$. The concatenated code $A \circ B$ is constructed in the following way: for any state $|\phi\rangle=\sum_{j_{1} \cdots j_{N}} \alpha_{j_{1} \cdots j_{N}}\left|j_{1} \cdots j_{N}\right\rangle$ of the outer code $A$, replace each basis vector $\left|j_{l}\right\rangle$ (where $j_{l}=0, \cdots, Q-1$ for $\left.l=1, \ldots, N\right)$ by a basis vector $\left|\psi_{j_{l}}\right\rangle$ of the inner code $B$. This mapping yields

$$
|\phi\rangle \mapsto \widetilde{\phi}\rangle=\sum_{j_{1} \cdots j_{N}} \alpha_{j_{1} \cdots j_{N}}\left|\psi_{j_{1}}\right\rangle \cdots\left|\psi_{j_{N}}\right\rangle,
$$

and the resultant code is an $\llbracket n N, k K, \mathcal{D} \rrbracket_{q}$ stabilizer code where $\mathcal{D} \geq d D$ [5], [8].

For GCQCs, the role of the basis vectors of the inner quantum code is taken on by subcodes of the inner code [3]. In its simplest version (two-level version), a GCQC is also constructed from two quantum codes: an outer code $A_{1}$ with with parameters $\llbracket N, K_{1}, D_{1} \rrbracket_{Q_{1}}$ and an inner code $B_{1}$ with parameters $\llbracket n, k_{1}, d_{1} \rrbracket_{q}$, such that the inner code $B_{1}$ could be

\footnotetext{
${ }^{1}$ In the sequel, we usually denote the outer parameters by capital Latin characters and the inner parameters by their small counterparts.
} 
further partitioned into $Q_{1}$ subcodes $\left\{B_{2}^{(j)}\right\}_{j=0}^{Q_{1}-1}$, i.e.,

$$
B_{1}=\bigoplus_{j=0}^{Q_{1}-1} B_{2}^{(j)}
$$

and each $B_{2}^{(j)}$ is an $\llbracket n, k_{2}, d_{2} \rrbracket_{q}$ code, with basis vectors $\left\{\left|\psi_{i}^{(j)}\right\rangle\right\}_{i=0}^{q^{k_{2}}-1}, j=0, \ldots, Q_{1}-1$ and $d_{2} \geq d_{1}$. Thus we have $q^{k_{1}-k_{2}}=Q_{1}$

To construct a GCQC, replace each basis state $|j\rangle$ of the outer code $A_{1}$ with a basis state $\left\{\left|\psi_{i}^{(j)}\right\rangle\right\}$ of $B_{2}^{(j)}$. In this way, each basis state $|j\rangle$ of the outer code is mapped to the subcode $B_{2}^{(j)}$. Consequently, given a state $|\phi\rangle=\sum_{j_{1} \cdots i_{N}} \alpha_{j_{1} \cdots j_{N}}\left|j_{1} \cdots j_{N}\right\rangle$ of the outer code together with an unencoded basis state $\left|i_{1} \cdots i_{N}\right\rangle \in$ $\left(\mathbb{C}^{q^{k_{2}}}\right)^{\otimes N}$, the encoding of a GCQC is given by the following mapping [3]:

$$
|\phi\rangle\left|i_{1} \cdots i_{N}\right\rangle \mapsto \sum_{j_{1} \cdots j_{N}} \alpha_{j_{1} \cdots j_{N}}\left|\psi_{i_{1}}^{\left(j_{1}\right)}\right\rangle \cdots\left|\psi_{i_{N}}^{\left(j_{N}\right)}\right\rangle .
$$

This then gives a GCQC code with parameters $\llbracket \mathcal{N}, \mathcal{K}, \mathcal{D} \rrbracket_{q}$, where $\mathcal{N}=n N, \mathcal{K}=\left(k_{1}-k_{2}\right) K_{1}+k_{2} N$, and the minimum distance $\mathcal{D}$ to be determined. Note that the basis states $\left|i_{1} \cdots i_{N}\right\rangle$ span a trivial outer code $\llbracket N, N, 1 \rrbracket_{Q_{2}}$, where $Q_{2}=q^{k_{2}}$. Therefore, two outer codes and two inner codes are used, which is where the name 'two-level concatenation' comes from.

\section{B. Quantum coset codes}

We adapt the concept of coset codes [1], [9], [10] to the quantum scenario to provide an alternative understanding for stabilizer GCQCs. Coset codes will help to build a systematic interpretation for GCQCs from the viewpoint of the stabilizer formalism.

We choose any subcode $B_{2}^{(j)}$ in the decomposition (2) and denote it as $B_{2}$. Continuing the partitioning process, say

$$
B_{i}=\bigoplus_{j=0}^{Q_{i}-1} B_{i+1}^{(j)}
$$

for $i=2,3, \ldots, m$, we obtain a chain of subcodes

$$
B_{m+1} \subset B_{m} \subset \cdots \subset B_{3} \subset B_{2} \subset B_{1},
$$

where all subcodes $B_{i}^{(j)}$ on level $i$ have parameters $\llbracket n, k_{i}, d_{i} \rrbracket_{q}$. To simplify notation, we use $B_{i}$ to denote any of the subcodes $B_{i}^{(j)}$. On level $m+1$, all subcodes are one-dimensional subspaces, and we choose $B_{m+1}=\{|\mathbf{0}\rangle\}$.

As the subspaces $B_{i+1}^{(j)}$ in the decomposition (4) are all isomorphic, we can, on an abstract level, rewrite the decomposition as a tensor product of a vector space of dimension $Q_{i}$, spanned by orthonormal states $|j\rangle$ corresponding to the indices $j$ in the decomposition (4), and the subcode $B_{i+1}$. We denote this situation by

$$
B_{i}=\llbracket B_{i} / B_{i+1} \rrbracket \otimes B_{i+1} .
$$

\footnotetext{
${ }^{2}$ The resultant code is reduced to the usual concatenated stabilizer code when $k_{2}=0$.
}

It turns out that the co-factor $\llbracket B_{i} / B_{i+1} \rrbracket$ in (6) can be identified with an additive quantum code of dimension

$$
Q_{i}=\operatorname{dim} \llbracket\left[B_{i} / B_{i+1} \rrbracket=q^{k_{i}-k_{i+1}} .\right.
$$

Note that both $B_{i+1}$ and $\llbracket B_{i} / B_{i+1} \rrbracket$ are defined with respect to a quantum system with $n$ qudits. In analogy to coset codes in the context of generalized concatenated codes [1], [9], [10], we call $\llbracket B_{i} / B_{i+1} \rrbracket$ a quantum coset code.

This then directly leads to

$$
B_{1}=\llbracket B_{1} / B_{2} \rrbracket \otimes \llbracket B_{2} / B_{3} \rrbracket \otimes \cdots \otimes B_{m},
$$

i. e., the quantum code $B_{1}$ is abstractly a tensor product of $m$ coset codes $\llbracket B_{1} / B_{2} \rrbracket, \llbracket B_{2} / B_{3} \rrbracket, \ldots, \llbracket B_{m} / B_{m+1} \rrbracket=B_{m}$. These $m$ quantum coset codes will be used as inner codes to be concatenated with $m$ outer codes $A_{i}(i=1,2, \ldots m)$ to form an $m$-level concatenated quantum code.

On each level, the basis state $|j\rangle \in \mathbb{C}^{Q_{i}}$ of the 'coordinate space' of the outer code $A_{i}=\llbracket N_{i}, K_{i}, D_{i} \rrbracket_{Q_{i}}$ is mapped to the basis index $j$ of the corresponding quantum coset code $\llbracket B_{i} / B_{i+1} \rrbracket$. Hence, the $i$ th level of concatenation yields the concatenated code

$$
C_{i}=A_{i} \circ \llbracket B_{i} / B_{i+1} \rrbracket .
$$

The resultant $m$-level concatenated code $C$ is then the abstract tensor product of those $m$ concatenated codes, i. e.,

$$
C=C_{1} \otimes C_{2} \otimes \cdots \otimes C_{m} .
$$

\section{STABILIZER FORMALISM FOR GENERALIZED CONCATENATED QUANTUM CODES}

\section{A. Stabilizers for the inner codes}

We now develop the stabilizer formalism for GCQCs based on the coset codes $\llbracket B_{i} / B_{i+1} \rrbracket$. For simplicity we consider the case $q=2$, i. e., all codes $B_{i}$ s are qubit stabilizer codes. The extension to larger dimensions $q$ is straightforward.

For the code $B_{1}=\llbracket n, k_{1}, d_{1} \rrbracket_{2}$, let $S_{B_{1}}=\left\{g_{1}, g_{2}, \ldots, g_{n-k_{1}}\right\}$ denote the set of generators of the stabilizer group. The corresponding sets of logical $X$ - and Z-operators for the $k_{1}$ encoded qubits are denoted by $\bar{X}_{B_{1}}=\left\{\bar{X}_{1}, \bar{X}_{2}, \ldots, \bar{X}_{k_{1}}\right\}$ and $\bar{Z}_{B_{1}}=\left\{\bar{Z}_{1}, \bar{Z}_{2}, \ldots, \bar{Z}_{k_{1}}\right\}$. Similarly, for the code $B_{i}$, we use $S_{B_{i}}$, $\bar{X}_{B_{i}}$, and $\bar{Z}_{B_{i}}$ to denote the set of stabilizer generators, the logical $X$-, and the logical $Z$-operators, respectively.

Note that $B_{i}=\llbracket n, k_{i}, d_{i} \rrbracket_{q}$ is a subcode of $B_{1}$, for $2 \leq i \leq$ $m+1$. Thus $S_{B_{i}}$ can be chosen as the union of $S_{B_{1}}$ and a set comprising $k_{1}-k_{i}$ commuting logical operators of $B_{1}$, which is denoted as $\hat{S}_{B_{i}}$. Without loss of generality, we choose $\hat{S}_{B_{i}}=$ $\left\{\bar{Z}_{1}, \bar{Z}_{2}, \ldots, \bar{Z}_{k_{1}-k_{i}}\right\}$. Thus we have

$$
\begin{aligned}
S_{B_{i}}=S_{B_{1}} \cup \hat{S}_{B_{i}} & =\left\{g_{1}, g_{2}, \ldots, g_{n-k_{1}}, \bar{Z}_{1}, \ldots, \bar{Z}_{k_{1}-k_{i}}\right\}, \\
\bar{Z}_{B_{i}} & =\left\{\bar{Z}_{k_{1}-k_{i}+1}, \bar{Z}_{k_{1}-k_{i}+2}, \ldots, \bar{Z}_{k_{1}}\right\}, \\
\bar{X}_{B_{i}} & =\left\{\bar{X}_{k_{1}-k_{i}+1}, \bar{X}_{k_{1}-k_{i}+2}, \ldots, \bar{X}_{k_{1}}\right\} .
\end{aligned}
$$

Note that eventually we will arrive at $B_{m+1}=\{|\overline{00 \cdots 0}\rangle\}$. This logical state $|\mathbf{0}\rangle$ is the only vector shared by all $B_{i}$.

Recall that the code $\llbracket B_{i} / B_{i+1} \rrbracket$ is an additive quantum code with dimension $Q_{i}=q^{k_{i}-k_{i+1}}$. Let $S_{\llbracket B_{i} / B_{i+1} \rrbracket}$ denote the 
set of generators of its stabilizer group. Defining the set $\tilde{S}_{B_{i+1}}=\bar{Z}_{B_{i+1}}=\left\{\bar{Z}_{k_{1}-k_{i+1}+1}, \bar{Z}_{k_{1}-k_{i+1}+2}, \ldots, \bar{Z}_{k_{1}}\right\}$, we have

$$
\begin{aligned}
S_{\llbracket B_{i} / B_{i+1} \rrbracket} & =S_{B_{i}} \cup \tilde{S}_{B_{i+1}} \\
& =\left\{g_{1}, \ldots, g_{n-k_{1}}, \bar{Z}_{1}, \ldots, \bar{Z}_{k_{1}-k_{i}}, \bar{Z}_{k_{1}-k_{i+1}+1}, \ldots, \bar{Z}_{k_{1}}\right\} .
\end{aligned}
$$

The logical operators of $\llbracket B_{i} / B_{i+1} \rrbracket$ are

$$
\text { and } \begin{aligned}
\bar{Z}_{\llbracket B_{i} / B_{i+1} \rrbracket} & =\left\{\bar{Z}_{k_{1}-k_{i}+1}, \bar{Z}_{k_{1}-k_{i}+2}, \ldots, \bar{Z}_{k_{1}-k_{i+1} / B_{i+1} \rrbracket}\right\} \\
\text { and } & =\left\{\bar{X}_{k_{1}-k_{i}+1}, \bar{X}_{k_{1}-k_{i}+2}, \ldots, \bar{X}_{k_{1}-k_{i+1}}\right\} .
\end{aligned}
$$

The structure is illustrated in Fig. 1 .

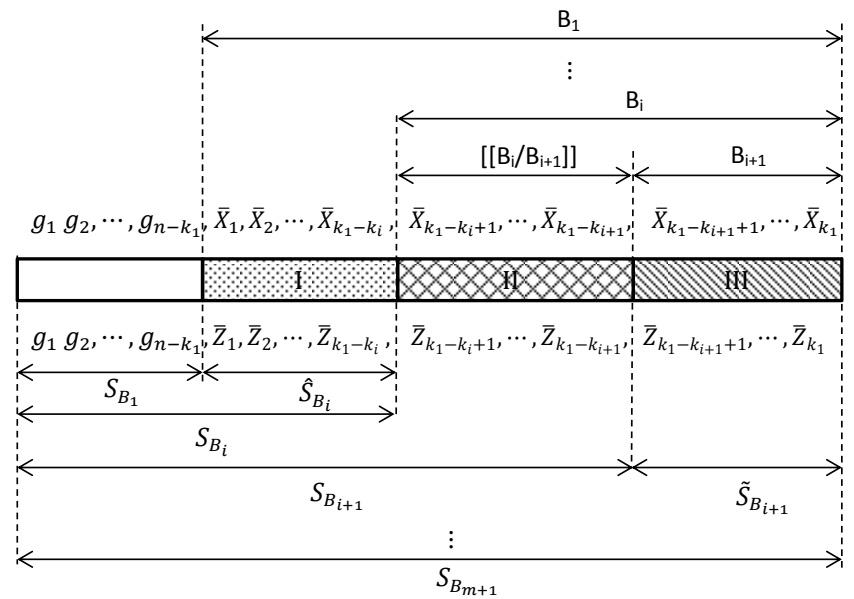

Fig. 1. Structure of a quantum coset code obtained by nesting $B_{i}$ s in computational basis. Here I, II, and III indicate the code factors $\llbracket B_{1} / B_{i} \rrbracket$, $\llbracket B_{i} / B_{i+1} \rrbracket$, and $B_{i+1}$, respectively. They are spanned by the states obtained when the corresponding logical operators located in their area act on the logical state $|\mathbf{0}\rangle$ shared by all subcodes $B_{i}$. All other terms are defined in the main text.

\section{B. Stabilizers for the generalized concatenated quantum codes}

We now discuss the stabilizers for a GCQCs with an inner code $B_{1}$ and its $m$-level partitions as given in Eq. (5). We will have $m$ outer codes $A_{i}, i=1,2, \ldots, m$, each with parameters $\llbracket N, K_{i}, D_{i} \rrbracket_{Q_{i}}$.

We first account for the stabilizer generators obtained solely from $B_{1}$. This set is denoted by $S_{I}$. The resulting GCQC has length $n N$. For each sub-block of length $n$, we have stabilizer generators from $S_{B_{1}}$ acting on that block. We can express $S_{I}$ as

$$
\begin{aligned}
& S_{I}=S_{B_{1}} \otimes\{\mathrm{id}\} \otimes\{\mathrm{id}\} \otimes \cdots \otimes\{\mathrm{id}\} \\
& \cup\{\mathrm{id}\} \otimes S_{B_{1}} \otimes\{\mathrm{id}\} \otimes \cdots \otimes\{\mathrm{id}\} \\
& \cup\{\mathrm{id}\} \otimes\{\mathrm{id}\} \otimes\{\mathrm{id}\} \otimes \cdots \otimes S_{B_{1}},
\end{aligned}
$$

where id denotes the identity operator on $n$ qubits, and the tensor product of two sets is defined as $S \otimes T=\{s \otimes t: s \in$ $S, t \in T\}$. Evidently, there are in total $\left(n-k_{1}\right) \times N$ independent generators in $S_{I}$.

Next we consider the contributions from the outer codes $A_{i}=\llbracket N, K_{i}, D_{i} \rrbracket_{Q_{i}}$. For $q=2$, we have $Q_{i}=2^{r_{i}}$, where $r_{i}=$ $k_{i}-k_{i+1}$. Each $A_{i}$ is a subspace of $\left(\mathbb{C}^{Q_{i}}\right)^{\otimes N} \cong\left(\mathbb{C}^{2}\right)^{\otimes r_{i} N}$. Hence each $A_{i}$ can be viewed as a subspace of $r_{i} N$ qubits which can be grouped into $N$ blocks with $r_{i}$ qubits in each block. Denote the set of generators for the stabilizer of $A_{i}$ by $S_{A_{i}}$. Any operator $G \in S_{A_{i}}$ can be expressed as $G=\bigotimes_{j=1}^{N} G_{j}$, where each $G_{j}=X^{\alpha} \cdot Z^{\beta}, \alpha, \beta \in G F\left(2^{r_{i}}\right)$ is a generalized Pauli operator on $\mathbb{C}^{2^{r_{i}}}$, which can be further represented as $\left(X^{a_{1}} X^{a_{2}} \cdots X^{a_{r_{i}}}\right) \cdot\left(Z^{b_{1}} Z^{b_{2}} \cdots Z^{b_{r_{i}}}\right)$, where $a_{j}, b_{j} \in G F(2)$ for $j=$ $1,2, \ldots, r_{i}[11]$

Note that each $X^{a_{\ell}}\left(Z^{b_{\ell}}\right)$ is a Pauli operator corresponding to the $\ell$ th qubit for each block with $r_{i}$ qubits. For the concatenation at the $i$ th level, each basis vector $|j\rangle$ of the 'coordinate space' of $A_{i}$ will be mapped to a basis vector $\left|b_{j}^{(i)}\right\rangle$ of the coset code $\llbracket B_{i} / B_{i+1} \rrbracket$. Therefore, in order to import the constraints coming from the stabilizer generators $S_{A_{i}}$, we need to replace the Pauli operators $X^{a_{l}}\left(Z^{b_{l}}\right)$ for each block of $r_{i}$ qubits by the corresponding logical operators of $\llbracket B_{i} / B_{i+1} \rrbracket$, which are given by Eqs. (15) and (16).

For each of the $N$ blocks in total, this procedure encodes $r_{i}$ qubits into $n$ qubits. For each $G_{j}=X^{\alpha} \cdot Z^{\beta},(1 \leq j \leq N)$, the replacement mentioned above yields

$$
\begin{aligned}
\overline{G_{j}}=( & \left.\bar{X}_{k_{1}-k_{i}+1}^{a_{1}} \bar{X}_{k_{1}-k_{i}+2}^{a_{2}} \cdots \bar{X}_{k_{1}-k_{i+1}}^{a_{r_{i}}}\right) \\
& \cdot\left(\bar{Z}_{k_{1}-k_{i}+1}^{b_{1}} \bar{Z}_{k_{1}-k_{i}+2}^{b_{2}} \cdots \bar{Z}_{k_{1}-k_{i+1}}^{b_{r_{i}}}\right) .
\end{aligned}
$$

Thus each generator $G \in S_{A_{i}}$ is mapped to $\bar{G}=\bigotimes_{j=1}^{N} \bar{G}_{j} \in$ $\bar{S}_{A_{i}}$, where $\bar{S}_{A_{i}}$ denotes the resulting set of generators after the replacement.

For each outer code $A_{i}$, denote the set of logical operators by $L_{A_{i}}$. Then using a similar replacement as for the stabilizer generators, we obtain a set of logical operators for the $i$ th level concatenated code, which we denote by $\bar{L}_{i}$. We then have the following proposition, which is a direct consequence of Eq. 10,.

Proposition 1. The set of stabilizer generator $S_{C}$ for the generalized concatenated quantum code $C=\llbracket \mathcal{N}, \mathcal{K}, \mathcal{D} \rrbracket_{q}$ is given by

$$
S_{C}=S_{I} \cup \bigcup_{i=1}^{m} \bar{S}_{A_{i}},
$$

and the set of logical operators $L_{C}$ is given by

$$
L_{C}=\bigcup_{i=1}^{m} \bar{L}_{i} \text {. }
$$

Note that we may multiply any logical operator by an element of the stabilizer without changing its effect on the code.

Example 2. Consider $B_{1}=\llbracket 4,2,2 \rrbracket_{2}$ with stabilizer generators $S_{B_{1}}=\{X X X X, Z Z Z Z\}$ and logical operators $\bar{Z}_{1}=Z Z I I$, $\bar{X}_{1}=X I X I, \bar{Z}_{2}=Z I Z I, \bar{X}_{2}=X X I I$. Then take the subcodes $B_{2}=\llbracket 4,1,2 \rrbracket_{2}$ with stabilizer generators $S_{B_{1}} \cup\left\{\bar{Z}_{1}\right\}$ and $B_{3}=\llbracket 4,0,2 \rrbracket_{2}$ with stabilizer generators by $S_{B_{1}} \cup\left\{\bar{Z}_{1}, \bar{Z}_{2}\right\}$. Thus the coset code $\llbracket B_{1} / B_{2} \rrbracket$ has dimension 2 with logical operators $\left\{\bar{Z}_{1}, \bar{X}_{1}\right\}$. It will be used as the inner code for the first level of concatenation. Since $B_{3}=\{|\mathbf{0}\rangle\}$, we have

\footnotetext{
${ }^{3}$ Here we omit the tensor product symbol, i.e., $X^{a_{1}} X^{a_{2}} \cdots X^{a_{r_{i}}}$ is to be read as $X^{a_{1}} \otimes X^{a_{2}} \otimes \cdots \otimes X^{a_{r_{i}}}$, similarly for $Z^{b_{1}} Z^{b_{2}} \cdots Z^{b_{r_{i}}}$.
} 
$\llbracket B_{2} / B_{3} \rrbracket \cong B_{2}$ with logical operators $\left\{\bar{Z}_{2}, \bar{X}_{2}\right\}$. It will be used as the inner code on the second level of concatenation.

For the outer codes, take $A_{1}=\llbracket 2,1,1 \rrbracket_{2}$ with stabilizer generators $S_{A_{1}}=\{Z Z\}$ and logical operators $\{Z I, X X\}$, together with the trivial code $A_{2}=\llbracket 2,2,1 \rrbracket_{2}$ with logical operators $\{Z I, X I, I Z, I X\}$. Then $\bar{S}_{A_{1}}=\left\{\bar{Z}_{1} \bar{Z}_{1}\right\}$, and the stabilizer $S_{C}$ of the resulting GCQC is thus generated by $S_{I} \cup \bar{S}_{A_{1}}$. Furthermore, the set of logical operators is given by $L_{C}=\left\{\bar{Z}_{1} I_{4}, \bar{X}_{1} \bar{X}_{1}, \bar{Z}_{2} I_{4}, \bar{X}_{2} I_{4}, I_{4} \bar{Z}_{2}, I_{4} \bar{X}_{2}\right\}$, where $I_{4}$ denotes the identity operator on each of the 4-qubit sub-blocks. The resulting GCQC has parameters $C=\llbracket 8,3,2 \rrbracket_{2}$.

\section{Parameters of GCQCs}

In order to derive the parameters of the GCQCs from our stabilizer formalism, we will use the following lemma. We keep the notation from the previous sections. In addition, for a stabilizer code with stabilizer generators $S$, we denote the normalizer group of $S$ by $N(S)$.

Lemma 3. Consider the restriction $\bar{W}_{\downarrow \bar{r}}$ and $\bar{V}_{\downarrow \bar{s}}$ of any two elements $\bar{W} \in N\left(\bar{S}_{A_{i}}\right)$ and $\bar{V} \in N\left(\bar{S}_{A_{j}}\right)(1 \leq i \leq j \leq m)$ to sub-block $\bar{r}$ and $\bar{s}(r, s \in\{1, \ldots, N\})$, respectively, each block corresponding to $n$ qubits obtained by mapping one coordinate of the outer code to the $n$ qubits of the inner code. Then the product $\bar{W}_{\downarrow \bar{r}} \cdot \bar{V}_{\downarrow \bar{s}}$ has weight at least $d_{i}$, unless $\bar{W}_{\downarrow \bar{r}}=\bar{V}_{\downarrow \bar{s}}=i d$.

Proof: Case 1: $i=j$ :

$\bar{W}_{\downarrow \bar{r}} \cdot \bar{V}_{\downarrow \bar{s}}$ is composed of the logical operators of $B_{i}$, whose distance is $d_{i}$, thus $\bar{W}_{\downarrow \bar{r}} \cdot \bar{V}_{\downarrow \bar{s}}$ has weight at least $d_{i}$.

Case 2: $i<j$ :

$\bar{W}_{\downarrow \bar{r}} \cdot \bar{V}_{\downarrow \bar{s}}$ is composed of the logical operators from $B_{i}$ and $B_{j}$. Further, $B_{j} \subset B_{i}$ implies $d_{j}>d_{i}$, thus $\bar{W}_{\downarrow \bar{r}} \cdot \bar{V}_{\downarrow \bar{s}}$ has weight at least $d_{i}$.

Theorem 4. Consider a GCQC $C=\llbracket \mathcal{N}, \mathcal{K}, \mathcal{D} \rrbracket_{q}$ which is composed of $m$ outer codes $A_{i}=\llbracket N, K_{i}, D_{i} \rrbracket_{Q_{i}}$ and $m$ inner codes $\llbracket B_{i} / B_{i+1} \rrbracket_{q}$ for $i=1,2, \ldots, m$, where the code $B_{i}=$ $\llbracket n, k_{i}, d_{i} \rrbracket_{q}$ is in the sub-code chain $B_{m+1} \subset B_{m} \subset \cdots \subset B_{2} \subset B_{1}$ and $Q_{i}=q^{r_{i}}=q^{k_{i}-k_{i+1}}$. Let $A_{\mu}$ be the first degenerate code regarding the ordering $A_{1}>A_{2}>\cdots>A_{m}$ of the outer codes. Then the parameters of $C$ are given as

1)

$$
\mathcal{N}=n N
$$

2)

$$
\mathcal{K}=\sum_{i=1}^{m}\left(k_{i}-k_{i+1}\right) K_{i}
$$

3)

$$
\mathcal{D} \geq \min \left\{d_{1} D_{1}, d_{2} D_{2}, \ldots, d_{\mu-1} D_{\mu-1}, d_{\mu} \min _{\mu \leq i \leq m}\left\{D_{i}\right\}\right\} .
$$

Note that if all outer codes are non-degenerate codes, it follows from Eq. 22, that

$$
\mathcal{D} \geq \min \left\{d_{1} D_{1}, d_{2} D_{2}, \ldots, d_{m} D_{m}\right\} .
$$

If the first outer code a is degenerate code, then

$$
\mathcal{D} \geq d_{1} \min _{1 \leq i \leq m}\left\{D_{i}\right\}
$$

Proof:

1) Eq. (20) is evidently true.

2) For each $A_{i}=\llbracket N, K_{i}, D_{i} \rrbracket_{Q_{i}}$, the number of independent generators in $S_{A_{i}}$ is $r_{i}\left(N-K_{i}\right)$, which is also the number of independent generators in $\bar{S}_{A_{i}}$. The number of independent generators in $S_{I}$ is equal to $\left(n-k_{1}\right) N$. Therefore, according to Proposition 1, we have

$$
\begin{aligned}
\mathcal{K} & =n N-\left(n-k_{1}\right) N-\sum_{i=1}^{m} r_{i}\left(N-K_{i}\right) \\
& =\sum_{i=1}^{m}\left(k_{i}-k_{i+1}\right) K_{i},
\end{aligned}
$$

where $r_{i}=k_{i}-k_{i+1}$ for $i=1,2, \ldots, m$.

3) For a stabilizer code with stabilizer $S$, the minimum distance is the minimum weight of an element in $N(S) \backslash S$. In other words, it is the minimum weight of non-trivial logical operators. We consider different cases how a logical operator of a QCQC can be composed according to Proposition 1 (see Fig. 2).

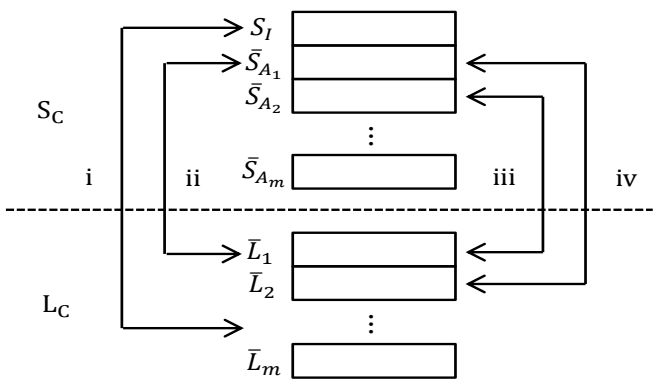

Fig. 2. Constitution of logical operators for a GCQC with all terms defined in the body.

For the $i$ th level of concatenation, we know that the distance of $\llbracket B_{i} / B_{i+1} \rrbracket$ is at least $d_{i}$. As the distance of $A_{i}$ is $D_{i}$, according to our replacement strategy, the non-trivial logical operators obtained from $\bar{L}_{i}$ and $\bar{S}_{A_{i}}$ have weight at least $d_{i}$ on at least $D_{i}$ sub-blocks of length $n$. Therefore, the minimal weight is at least $d_{i} D_{i}$. Multiplying two non-trivial elements $\bar{l}_{i}$ and $\bar{l}_{j}$ from two different levels $i$ and $j$ with $i<j$, from Lemma 3 the product $\bar{l}_{i j}=\bar{l}_{i} \cdot \bar{l}_{j}$ must have weight at least $d_{i}$ on at least $D_{i}$ sub-blocks of length $n$. Denoting the weight of an operator $\bar{l}$ as $\operatorname{wgt}(\bar{l})$, for any element $\bar{l} \in L_{C}$ (see Eq. (19)), we have

$$
\operatorname{wgt}(\bar{l}) \geq \min \left\{d_{1} D_{1}, d_{2} D_{2}, \ldots, d_{m} D_{m}\right\}
$$

Next we consider the minimal weight of the elements obtained by multiplying a logical operator $\bar{l} \in L_{C}$ by a nontrivial stabilizer element $\bar{G} \in S_{C}$. First let $\bar{l}^{\prime}=\bar{G} \cdot \bar{l}_{i}$, where $\bar{G} \in S_{I}$ or $\bar{G} \in \bar{S}_{A_{j}}$, and $\bar{l}_{i} \in \bar{L}_{i}$ for $1 \leq i, j \leq m$. Then we analyze $\operatorname{wgt}\left(\bar{l}^{\prime}\right)$ based on the following cases (see Fig. (2):

(i) $\bar{G} \in S_{I}$ : $\operatorname{wgt}\left(\bar{l}^{\prime}\right) \geq d_{i} D_{i}$ according to Eq. (5).

(ii) $\bar{G} \in \bar{S}_{A_{j}}$ and $i=j: \operatorname{wgt}\left(\bar{l}^{\prime}\right) \geq d_{i} D_{i}$.

(iii) $\bar{G} \in \bar{S}_{A_{j}}$ and $i<j$ : $\operatorname{wgt}\left(\bar{l}^{\prime}\right) \geq d_{i} D_{i}$ according to Lemma3, 
(iv) $\bar{G} \in \bar{S}_{A_{j}}$ and $i>j: \operatorname{wgt}\left(\bar{l}^{\prime}\right) \geq d_{j} \times \max \left\{D_{i}, \operatorname{wgt}(G)\right\}$ according to Lemma 3 If $A_{j}$ is a non-degenerate outer code, then $\operatorname{wgt}(G) \geq D_{j}$, thus $\operatorname{wgt}\left(\bar{l}^{\prime}\right) \geq d_{j} D_{j}$. If $A_{j}$ is a degenerate outer code, then there exist at least one nontrivial element $G \in S_{A_{j}}$ such that $\operatorname{wgt}(G)<D_{j}$, consider $D_{i}<D_{j}$ is probably true, thus $\operatorname{wgt}\left(\bar{l}^{\prime}\right) \geq d_{j} D_{j}$ is not guaranteed, but $\operatorname{wgt}\left(\bar{l}^{\prime}\right) \geq d_{j} D_{i}$ evidently is.

Any non-trivial logical operator can be decomposed as a combination of the four cases discussed above. Now we are ready to get the distance of a GCQC as shown by Eqs. (22), (23), and (24).

Note that a degenerate outer code might also be viewed as a non-degenerate code, but with a smaller distance. As clarified by Theorem 4 and illustrated by the following example, despite the larger minimum distance of the degenerate outer code, the minimum distance of the resulting GCQC is not increased in general.

Example 5. Let $B_{1}=\llbracket 4,2,1 \rrbracket_{2}$ with stabilizer generators $S_{B_{1}}=\{Z Z Z Z, Z Z I I\}$ and logical operators $\left\{\bar{Z}_{1}=X X X X, \bar{X}_{1}=\right.$ $\left.I Z Z Z, \bar{Z}_{2}=I I X X, \bar{X}_{2}=I Z I Z\right\}$. The subcodes $B_{1}$ and $B_{2}$ are $B_{2}=\llbracket 4,1,2 \rrbracket_{2}$ with stabilizer generators $S_{B_{1}} \cup\left\{\bar{Z}_{1}\right\}$, and $B_{3}=\llbracket 4,0,2 \rrbracket_{2}$ with stabilizer generators $S_{B_{1}} \cup\left\{\bar{Z}_{1}, \bar{Z}_{2}\right\}$. Then $\llbracket B_{1} / B_{2} \rrbracket$ of dimension 2 with logical operators $\left\{\bar{Z}_{B_{1}}, \bar{X}_{B_{1}}\right\}$, is the inner code to be used on the first level of concatenation, and $\llbracket B_{2} / B_{3} \rrbracket \cong B_{2}$ with logical operators $\left\{\bar{Z}_{2}, \bar{X}_{2}\right\}$ is the inner code to be used for the second level of concatenation.

The outer code $A_{1}=\llbracket\left[5,1,2 \rrbracket_{2}\right.$ is a degenerate code with stabilizer generators $S_{A_{1}}=\{X I I I I, I X X X X, I Z Z Z Z, I I I Z Z\}$ and logical operators $\{I Z I Z I, I X X I I\}$. Furthermore, let $A_{2}=\llbracket 5,5,1 \rrbracket_{2}$ be the trivial code with logical operators $\{Z I I I I, X I I I I, I Z I I I, I X I I I, \ldots, I I I I Z, I I I I X\}$. According to our replacement strategy, $\bar{G}=\bar{X}_{1} I_{4} I_{4} I_{4} I_{4} \in \bar{S}_{A_{1}}$ and $\bar{l}=\bar{X}_{2} I_{4} I_{4} I_{4} I_{4} \in \bar{L}_{2}$. Note that the minimum weight of elements in $\bar{L}_{2}$ is 2 , and $\operatorname{wgt}(\bar{l})=2$. Now consider the product $\bar{G} \cdot \bar{l}$ which is obviously a logical operator of the resulting GCQC as well and which plays the same role as $\bar{l}$. It is easy to check that multiplication by $\bar{G}$ reduces the weight of this logical operator from $d_{2} D_{2}=2$ to $d_{1} D_{2}=1$, as predicted by Eqs. (22) and (24).

In fact, $A_{1}$ can also be viewed as a non-degenerate code with parameters $\llbracket 5,1,1 \rrbracket_{2}$. This gives the lower bound $\operatorname{wgt}(G) \geq$ $D_{1}$, and therefore $\operatorname{wgt}(\bar{G} \cdot \bar{l}) \geq d_{1} \times \max \left\{D_{2}, D_{1}\right\} \geq d_{1} D_{1}=1$, which is consistent with Eq. (23). In summary, the resultant GCQC has parameters $\llbracket 20,6,1 \rrbracket_{2}$, but not $\llbracket 20,6,2 \rrbracket_{2}$ as one would expect for non-degenerate codes.

\section{Discussion}

We have developed the structure of the stabilizer and logical operators of generalized concatenated quantum codes. With the help of quantum coset codes $\llbracket B_{i} / B_{i+1} \rrbracket$, the resulting code can be considered as an abstract tensor product of codes $C_{i}$ corresponding to the $i$ th level of concatenation. For the code $C_{i}$, the lower bound on the minimum distance is $d_{i} D_{i}$. This lower bound is met only if all the non-identity entries of some logical operator of minimum weight $D_{i}$ of $A_{i}$ are mapped onto the logical operators of minimum weight $d_{i}$ of $\llbracket B_{i} / B_{i+1} \rrbracket$. In some cases, it is possible to use a clever map to improve the minimum distance of $C_{i}$ and thereby that of the resulting code.

Example 6. Take both $A_{1}$ and $B_{1}$ as $\llbracket 2,1,1 \rrbracket_{2}$ with stabilizer generator $\{Z Z\}$ and logical operators $\{\bar{Z}=Z I, \bar{X}=X X\}$. Take $B_{2}=\{|00\rangle\}$ as the trivial one-dimensional code. Then $\llbracket B_{1} / B_{2} \rrbracket \cong B_{1}$ with logical operators $\left\{\bar{Z}_{1}=Z I, \bar{X}_{1}=X X\right\}$. Now we swap the role of the logical $X$ - and the logical $Z$-operator of $\llbracket B_{1} / B_{2} \rrbracket$. In other words, we let $\bar{Z}_{1}^{\prime}=X X$, $\bar{X}_{1}^{\prime}=Z I$. Then according to our replacement strategy, we obtain a concatenated code $\llbracket 4,1,2 \mathbb{1}_{2}$ with stabilizer generators $\{Z Z I I, I I Z Z, X X X X\}$ and logical operators $\{X X I I, Z I Z I\}$, while the original choice of logical operators for $B_{1}$ would only give a code $\llbracket 4,1,1 \rrbracket_{2}$.

This example indicates that the minimum distance of the resulting GCQC might be significantly improved compared to the lower bound when a deliberate nesting strategy is used. That is because such a strategy could be used to optimize the weight distribution for the logical operators of the inner code $B_{1}$. The stabilizer of the quantum coset codes $\llbracket B_{i} / B_{i+1} \rrbracket$ depends on this choice, and hence the parameters of the inner codes as well. In combination with suitable chosen outer codes, the error-correcting capacity of component codes could be exploited efficiently and the overall performance might be better.

\section{ACKNOWLEDGMENT}

YJW is supported by NSERC, AITF and RFDP. The CQT is funded by the Singapore MoE and the NRF as part of the Research Centres of Excellence programme. BZ and BCS are each supported by NSERC and CIFAR.

\section{REFERENCES}

[1] S. Lin and D. J. Costello, Error Control Coding: Fundamentals and Applications 2nd ed., Pearson-Prentice Hall, New Jersey, 2004.

[2] I. Dumer, "Concatenated Codes and Their Multilevel Generalizations," in Handbook of Coding Theory, V.S. Pless and W. C. Huffman, eds. Elsevier, Amsterdam, 1998, ch. 23, pp. 1911-1988.

[3] M. Grassl, P. Shor, G. Smith, J. Smolin, and B. Zeng, "Generalized concatenated quantum codes," Physical Review A, vol. 79, no. 5, 050306, 2009.

[4] M. Grassl, P. Shor and B. Zeng, "Generalized concatenation for quantum codes," in Proceedings 2009 IEEE International Symposium on Information Theory (ISIT 2009), Seoul, Korea Jul. 2009, pp. 953-957.

[5] D. Gottesman, "Stabilizer codes and quantum error correction," $\mathrm{Ph} . \mathrm{D}$ thesis, California Institute of Technology, Pasadena, CA 1997.

[6] A. Calderbank, E. Rains, P. Shor, and N. Sloane, "Quantum error correction via codes over GF(4)," IEEE Trans. Inform. Theory, vol. 44, no. 4, pp. 1369-1387, July 1998.

[7] M. A. Nielsen, and I. L. Chuang, Quantum Computation and Quantum Information, Cambridge University Press, Cambridge, 2000.

[8] E. Knill, and R. Laflamme, "Concatenated Quantum Codes", quant-ph/9608012

[9] D. Forney, "Coset codes - Part I: Introduction and geometrical classification", IEEE Trans. Inform. Theory, vol. 34, no. 5, pp. 1123-1151, September 1988.

[10] D. Forney, "Coset codes - Part II: Binary lattices and related codes", IEEE Trans. Inform. Theory, vol. 34, no. 5, pp. 1152-1187, September 1988.

[11] A. Ashikhmin and E. Knill, "Nonbinary quantum stabilizer codes," IEEE Trans. Inform. Theory, vol. 47, no. 7, pp. 3065-3072, November 2001. 